\title{
The role of immunohistochemical staining with CK7 in the differential diagnosis of autoimmune hepatitis
}

\author{
Ozsen $\mathrm{M}^{1}$, Adim $\mathrm{SB}^{2}$, Akyildiz EU², Gurel $\mathrm{S}^{3}$ \\ Department of Pathology, Erzurum Regional Training and Research Hospital, Erzurum, Turkey. \\ m.isikoglu@hotmail.com
}

\begin{abstract}
INTRODUCTION: Although the immunohistochemical staining with cytokeratin 7 (CK7) is an adjuvant method for identifying various components of the intrahepatic biliary system, the expression of CK7 does not occur in hepatocytes. In the literature, some studies suggest that a group of cells having dual morphologic and immunophenotypic characteristics of bile duct epithelium and hepatocytes, referred to as progenitor stem cells, was stained positive with CK7.

MATERIALS AND METHODS: In this study, we examined a total of 219 cases diagnosed with autoimmune hepatitis, chronic hepatitis B, chronic hepatitis C, and primary biliary cholangitis between 2005 and 2017 in Uludağ University, Faculty of Medicine, Department of Medical Pathology.

RESULTS: The comparisons of AIH cases with HepB, HepC and PBC cases demonstrated that the immunoreactivity to CK7 was significantly higher in the AlH group $(p<0.005)$ compared to the groups of HepB and HepC, whereas no significant differences were found between the AlH and PBC groups. CONCLUSIONS: In our study, it was concluded that the immunoreactivity to CK7 could be used as an adjuvant treatment to the clinicopathologic assessment in distinguishing between the AlH cases and chronic viral hepatitis. However, since CK7 immunoreactive hepatocytes were widely detected also in patients with chronic viral hepatitis, and there was no statistically significant difference between the PBC and AlH cases, it has been established that the inclusion of CK7 immunoreactivity into the diagnostic histopathological criteria for AlH would not be convenient (Tab. 1, Fig. 1, Ref. 22). Text in PDF www.elis.sk. KEY WORDS: CK7, ductular hepatocyte, autoimmune hepatitis, progenitor stem cells.
\end{abstract}

\section{Introduction}

Autoimmune hepatitis (AIH) is a rare chronic inflammatory disease of the liver, which was first identified by Jan Waldenström in 1951 (1). Studies conducted in both Europe and the United States reported that the incidence was 1 to 2 , and the prevalence was 11 to 17 per 100,000 people; the diagnosis and treatment of the disease is of clinical importance since it would become an important cause of morbidity and mortality if not treated (2).

Several studies have revealed the presence of cells in the liver that have common characteristics of hepatocytes and biliary epithelial cells, and have intermediate features called "ductal hepatocytes". These cells were shown to be similar to progenitor stem cell populations using various types of immunohistochemical staining, mainly CK7, and can be seen in various conditions such as autoimmune liver diseases, chronic biliary diseases, and chronic hepatitis B (3-5).

${ }^{1}$ Department of Pathology, Erzurum Regional Training and Research Hospital, Erzurum, Turkey, ${ }^{2}$ Department of Pathology, Uludag University Faculty of Medicine, Bursa, Turkey, and ${ }^{3}$ Department of Gastroenterology, Uludag University Faculty of Medicine, Bursa, Turkey

Address for correspondence: M. Ozsen, Erzurum Regional Training and Research Hospital Department of Pathology, Erzurum, Turkey.

Phone: +532.1601737 , Fax: +224.2950019
The aim of this study was to demonstrate any change in periportal cells, referred to as progenitor cells leading to hyperplasia, using immunohistochemical staining with $\mathrm{CK} 7$ in patients diagnosed with $\mathrm{AIH}$, as well as to investigate the importance of progenitor cells leading to hyperplasia for making the differential diagnosis in chronic hepatitis cases, and look into its potential use as an objective criterion for the diagnosis of $\mathrm{AIH}$.

\section{Materials and methods}

We enrolled the patients diagnosed with autoimmune hepatitis $(\mathrm{AIH})$, hepatitis $\mathrm{B}(\mathrm{HepB})$, hepatitis $\mathrm{C}(\mathrm{HepC})$, and primary biliary cholangitis (PBC) between 2005 and 2017 in Uludag University, Faculty of Medicine, Department of Medical Pathology. Patients with unavailable clinical-laboratory data, overlap syndromes, or poor follow-up quality were excluded from the study. Age, gender, clinical and laboratory data were obtained from the electronic database.

Hematoxylin-eosin (HE)-stained sections from the patients included in the study were re-evaluated in terms of histopathological diagnosis, interphase activity and histopathological parameters such as fibrosis. "ISHAK Modified Histological Activity Index Grading Scale" and "ISHAK Fibrosis Scoring System” were used in the evaluation of the interphase activity and fibrosis, respectively. 

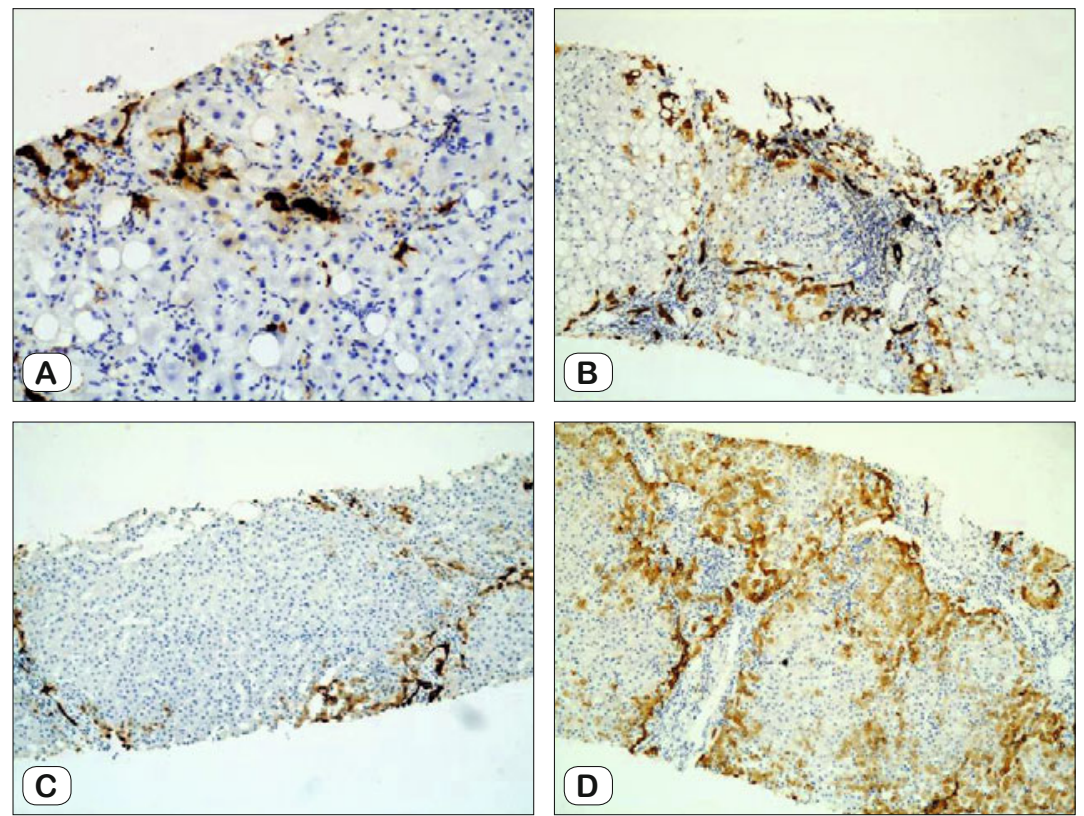

Fig. 1. A) CK7, positive staining (Staining Score: 1) (x100), B) CK7, positive staining (Staining score: 2) (x40), C) CK7, positive staining (Staining Score: 3) (x40), D) CK7, positive staining (Staining Score: 4$)$ (x100).

For immunohistochemical staining, the most suitable preparations for evaluation of each case were selected, and their paraffin blocks were obtained from the pathology archive. For immunohistochemical staining with CK7, 4-micrometer-thick sections were cut from these blocks, and then placed on positively charged slides. Liver tissue was used as control block for CK7 antibody. The cytoplasmic staining of CK7 antibody in hepatocytes was considered immunoreactive, and the staining with CK7 was evaluated in two different ways. In the first evaluation, the values assigned were based on the presence and intensity of antibody staining as follows: negative for no staining; 1 - positive for any focal staining in hepatocytes surrounding a portal area; 2 - positive for any focal staining in hepatocytes surrounding multiple portal areas; 3 - positive for any diffuse staining in hepatocytes surrounding multiple portal areas; and 4 - positive for any staining of hepatocytes including those in zones 2 and 3 (Fig. 1). In the second evaluation, the assignments were performed based on the presence of antibody staining as follows: negative for no staining in hepatocytes, positive for staining of hepatocytes in any location.

\section{Statistical analysis}

The statistical analyses of the results were performed using SPSS statistical software package (IBM Corp. Released 2012. IBM SPSS Statistics for Windows, Version 21.0. Armonk, NY: IBM Corp.) by the Department of Biostatistics, Uludağ University. Based on the results of normality test, Mann-Whitney test with two independent samples was used for comparison between two groups, whereas Kruskall Wallis test with independent samples was used for comparison between four groups, and Pearson's chi-square test was applied for intergroup comparison of categorical variables. $P$ values less than 0.05 were considered statistically significant. Since there was no statistically significant difference between the two different evaluation systems used to rate CK7 immunoreactivity, the second evaluation system was employed.

The approval for this study was obtained from Uludağ University, Faculty of Medicine, Ethics Committee of Medical Research, dated 03 October 2017 and numbered 2017$14 / 50$.

\section{Results}

Of the 219 patients diagnosed with $\mathrm{AIH}$, HepB, HepC, or PBC, 153 (69.8 \%) were female and $66(30.2 \%)$ were male. The ratio of females to males was found to be 2.31 . The mean age of patients was 46.6 years (ranging from 5 to 76), while the mean age of men was 43.8 years (ranging from 11 to 76 ) and the mean age of women was 47.8 years (ranging from 5 to 74).

Of the 219 patients, $64(29.2 \%)$ were diagnosed with AIH, 63 (28.8 \%) with HepB, 62 (28.3 \%) with HepC, and 30 (13.7\%) with $\mathrm{PBC}$.

The interphase activity was found to be 0 in 49 patients $(22.4 \%), 1$ in 38 patients $(17.4 \%), 2$ in 46 patients $(21 \%), 3$ in 36 patients $(16.4 \%)$, and 4 in 50 patients $(22.8 \%)$. There were no cases with the interphase activity of 5 .

The degree of fibrosis of 0 was found in 88 patients (40.2\%), 1 in 23 patients $(10.5 \%), 2$ in 39 patients $(17.8 \%), 3$ in 31 patients $(14.1 \%), 4$ in 18 patients $(8.2 \%), 5$ in 14 patients $(6.4 \%)$, and 6 in 6 patients $(2.8 \%)$.

The immunohistochemical analysis using CK7 antibody revealed that 113 of the 219 patients $(51.6 \%)$ had immunoreactivity, whereas 106 patients $(48.4 \%$ ) had no immunoreactivity (Tab. 1). The proportion of those with positive CK7 immunoreactivity to the total number of cases was $21 \%$ for AIH, $6.4 \%$ for HepB, $12.3 \%$ for HepC, and $8.7 \%$ for PBC. The immunoreactivity to CK 7 was

Tab. 1. Clinicopathological data of patients based on CK7 staining characteristics.

\begin{tabular}{lccc}
\hline & $\begin{array}{c}\text { Negative } \\
(\mathrm{n}=113)\end{array}$ & $\begin{array}{c}\text { Positive } \\
(\mathrm{n}=106)\end{array}$ & $\mathrm{p}$ \\
\hline Age & $42.07(5-72)$ & $51.5(12-76)$ & $0.123^{*}$ \\
Gender (F/M) & $75 / 39$ & $82 / 27$ & $<0.001^{*}$ \\
ANA(+) & 22 & 52 & $<0.001^{*}$ \\
AMA(+) & 11 & 24 & $0.019^{*}$ \\
AMA M2(+) & 9 & 28 & $0.001^{*}$ \\
IgG & 8 & 38 & $<0.001^{*}$ \\
IgM & 8 & 23 & $0.004^{*}$ \\
\hline
\end{tabular}

* Pearson Chi Square test 
significantly higher in the AIH group $(\mathrm{p}<0.001)$ compared to the HepB group. A similar result was obtained from the comparison of the former group with HepC cases. The positive immunoreactivity to CK7 was also significantly higher in the AIH patients ( $\mathrm{p}$ $<0.001$ ) compared to those diagnosed with the HepC. There was no statistically significant difference between the AIH and PBC cases in terms of CK7 immunoreactivity.

Again, no statistically significant difference was found between the HepB and HepC cases regarding CK7 immunoreactivity.

When PBS and HepB cases were compared in terms of CK7 immunoreactivity, the percentage of CK7 positive staining in $\mathrm{PBC}$ cases was found to be statistically significantly higher than HepB cases $(p<0.001)$. However, no statistically significant difference was found between PBC and HepC cases.

When examining the relationship between $\mathrm{CK} 7$ immunoreactivity and interphase activity, it was also shown that the likelihood of staining with CK7 increased with higher interphase activity ( $p$ $<0.001$ ). On the other hand, the relationship between CK7 immunoreactivity and fibrosis degree was non-linear but statistically significant $(\mathrm{p}<0.001)$.

Of the cases with CK 7 immunoreactivity, ANA positivity was detected in 51 cases, whereas AMA positivity and AMA M2 positivity were present in 23 and 27 cases, respectively. Although the relationship between $\mathrm{CK} 7$ immunoreactivity and ANA positivity was found to be statistically significant ( $p<0.001$ ), this was not the case between AMA and AMA M2.

The levels of $\operatorname{IgG}$ and IgM were found to be higher in 38 and 23 cases with CK7 immunoreactivity, respectively. There was a statistically significant correlation between CK7 immunoreactivity and elevated levels of $\operatorname{IgG}$ and $\operatorname{IgM}(p<0.001$ and $p<0.005$, respectively). The relationship between IgA level and CK7 immunoreactivity could not be evaluated due to the lack of sufficient data.

\section{Discussion}

Initially denominated as lupoid hepatitis due to its relationship with autoimmune conditions, and detection of lupus erythematosus cells, the term AIH has been used since Mackay et al identified the current notion of autoimmune hepatitis in 1995 (6).

Although the AIH can be detected in both genders and in any age group, its prevalence is known to be four times higher in women than in men. It may cause a bimodal age distribution in women, including early adulthood and period of menopause (2, 7-9). Of all AIH patients in our study, 56 were female (87.5\%) and 8 were male $(12.5 \%)$. The female/male ratio of $7: 1$ in our series was higher than those reported in the literature. The mean age at diagnosis of our AIH patients was 45.34 years, ranging from 5 to 74 years. The diagnosis of AIH cannot be made easily, and as such should be based on a combination of clinical presentation, biochemical liver tests, serological findings such as serum IgG and autoantibodies, presence of viral hepatitis, and histopathological findings (10-12). Although not necessary for making the diagnosis, biopsy alone is not a sufficient criterion. The presence of interphase hepatitis strongly supports the diagnosis of AIH since its histopathological finding occurs in 84 to $98 \%$ of all AIH cases. However, it can also occur in other types of hepatitis developing for different reasons (12). In our study, when we compared the cases in terms of interphase activity, we found that interphase activity was significantly lower in HepB cases compared to that in AIH cases $(p<0.05)$. There was no significant difference between the cases of HepC and PBC, and AIH cases.

Although not contributing to the diagnosis of $\mathrm{AIH}$, fibrosis is a key histopathological finding in terms of guiding the treatment (13). In our study, when we compared the cases in terms of fibrosis, we found that the degree of fibrosis was significantly lower in HepB cases compared to that in AIH cases $(p<0.05)$. No significant difference was detected between the cases of HepC and $\mathrm{PBC}$, and $\mathrm{AIH}$ cases.

As is known, the liver is an organ highly capable of regeneration especially in case of mild-to-moderate damage which occurred due to various etiologies. In cases of such damage, the main regeneration mechanism is based on the proliferation ability of hepatocytes. The general belief that the proliferation of hepatocytes alone is not enough for regeneration in serious and chronic damage, gave rise to the need for further studies in order to understand the development and regeneration of the liver. These studies, which had been conducted on animal models for many years, have also provided the basis of human studies (14-16).

A group of cells involved in liver regeneration has been identified in studies on animal models. Having dual morphological and immunophenotypical characteristics of the bile duct epithelium and hepatocytes, these cells are called oval cells in animals and hepatic progenitor cells in humans. They are thought to originate from Hering's canals, and studies have shown that they can also be seen in autoimmune liver diseases, chronic biliary diseases and chronic viral hepatitis $(3,17-20)$.

In a healthy human liver, hepatocytes are immunohistochemically expressed with CK8 and CK18, but not with CK7. It is known that ductular hepatocytes can be detected as hepatocytes with abnormal CK7 expression in some liver diseases such as viral hepatitis, PBC, and PSC, especially in the periportal region $(3,14,21)$. The fact that the diagnosis of AIH is challenging, and cholestasis is more dominant in autoimmune liver diseases compared to chronic viral hepatitis has led to the idea that the presence of hepatic progenitor cells may contribute to the differential diagnosis between viral hepatitis and autoimmune liver diseases.

In their study of 113 patients with HepB, HepC, nonalcoholic liver disease, $\mathrm{PBC}, \mathrm{AIH}$, and other non-neoplastic liver diseases due to various etiologies, Matsukuma et al. have found that hepatocytes were immunohistochemically stained with CK7 in $56 \%$ of the cases. While CK7 expression was observed in $57.1 \%$ of PBC, $50 \%$ of HepC, and $40 \%$ of AIH cases, it was $38.1 \%$ in HepB cases (14).

Goldstein et al investigated the role of CK 7 expression in cases with early stage of differentiation in mildly active $\mathrm{PBC}$ cases and AIH cases. In their study, the mean CK7-reactive periportal hepatocyte scores in the biopsy specimens of PBC and AIH cases were 2.13 (ranging from 0 to 3 ) and 0.13 (ranging from 0 to 1 ), 
839-842

respectively. In this study, where CK7 expression was scored between 0 and 3, the periportal CK 7 hepatocyte score was either 0 or 1 in $7(35 \%)$ of the $20 \mathrm{PBC}$ cases and all $(100 \%)$ of the $18 \mathrm{AIH}$ cases. None of the AIH cases had CK7 expression score above 1 (22). In our study, the mean CK7-immunoreactive hepatocyte score was 1.797 in the biopsy specimens of AIH cases, whereas it was 0.413 in the HepB cases, 1.048 in the HepC cases and 1.9 in the PBC cases. While CK7-immunoreactive hepatocyte score was found to be significantly higher in the AIH cases compared to those of HepB and HepC cases $(p<0.005)$, no significant difference was observed with PBC cases $(p>0.005)$.

To the best of our knowledge, the CK7 immunoreactivity can be used as an adjuvant method to clinicopathologic assessment in distinguishing between the cases of AIH and chronic viral hepatitis. However, since CK7 immunoreactive hepatocytes were widely detected also in patients with chronic viral hepatitis, and there was no statistically significant difference between PBC cases and AIH cases, it has been established that the inclusion of CK7 immunoreactivity into the diagnostic histopathological criteria for $\mathrm{AIH}$ would not be convenient.

\section{References}

1. Waldenstrom J. Liver, blood proteins and food proteins. Dtsch Z Verdau Stoffwecheselkr 1952; 30 (2): 803-807.

2. Manns MP, Czaja AJ, Gorham JD et al. Diagnosis and management of autoimmune hepatitis. Hepatology 2010; 51: 2193-2213.

3. Tan J, Hytiroglou P, Wieczorek R et al. Immunohistochemical evidence for hepatic progenitor cells in liver diseases. Liver 2002; 22 (5): 365-373.

4. Portmann B, Popper H, Neuberger J, Williams R. Sequential and diagnostic features in primary biliary cirrhosis based on serial histologic study in 209 patients. Gastroenterology 1985; 88: 1777-1790.

5. Barakauskienė A, Speičienė $D$, Liakina $V$ et al. Expression of cytokeratin 7 as a histological marker of cholestasis and stages of primary biliary cirrhosis. Send to Medicina (Kaunas) 2011; 47 (1): 31-38.

6. Mackay IR, Weiden S, Hasker J. Autoimmune hepatitis. Ann N Y Acad Sci 1965; 124: 767-780.

7. Al-Chalabi T, Boccato S, Portmann BC et al. Autoimmune hepatitis (AIH) in the elderly: a systematic retrospective analysis of a large group of consecutive patients with definite AIH followed at a tertiary referral centre. Journal of hepatology 2006; 45 (4): 575-583.
8. Van Gerven NM, Verwer BJ, Witte BI et al. Dutch Autoimmune hepatitis STUDY group. Epidemiology and clinical characteristics of autoimmune hepatitis in the Netherlands. Scand J Gastroenterol 2014; 49 (10): 1245-1254.

9. Gatselis NK, Zachou K, Koukoulis GK et al. Autoimmune hepatitis, one disease with many faces: etiopathogenetic, clinico-laboratory and histological characteristics. World J Gastroenterol 2015; 21 (1): 60-83.

10. Lohse AW, Mieli-Vergani G. Autoimmune hepatitis. J Hepatol 2011; 55 (1): 171-182.

11. Sahebjam F, Vierling JM. Autoimmune hepatitis. Front Med 2015; 9 (2): 187-219. doi: 10.1007/s11684-015-0386-y.

12. Van Gerven NM, De Boer YS, Mulder CJ et al. Autoimmune hepatitis. World J Gastroenterol 2016; 22 (19): 4651-4661.

13. Abe M, Mashiba T, Zeniya M et al; Autoimmune Hepatitis Study Group-Subgroup of the Intractable Hepato-Biliary Disease Study Group in Japan. Present status of autoimmune hepatitis in Japan: a nationwide survey. J Gastroenterol 2011; 46 (9): 1136-1141.

14. Matsukuma S, Takeo H, Kona T et al. Aberrant cytokeratin 7 expression of centrilobular hepatocytes: a clinicopathological study. Histopathology 2012; 61 (5): 857-862.

15. Lu WY, Bird TG, Boulter $L$ et al. Hepatic progenitor cells of biliary origin with liver repopulation capacity. Nat Cell Biol 2015; 17 (8): 971-983. doi: 10.1038/ncb3203.

16. Villano G, Turato C, Quarta S et al. Hepatic progenitor cells express SerpinB3. BMC Cell Biol 2014 Feb 11; 15: 5. doi: 10.1186/1471-2121-15-5.

17. Fausto N. Liver regeneration and repair: hepatocytes, progenitor cells, and stem cells. Hepatology 2004; 39 (6): 1477-1487.

18. Roskams T. Different types of liver progenitor cells and their niches. J Hepatol 2006; 15: 1-4. doi: 10.1016/j.jhep.2006.05.002.

19. Kordes C, Häussinger D. Hepatic stem cell niches. J Clin Invest 2013; 15: 1874-1880. doi: 10.1172/JCI66027.

20. Roskams TA, Theise ND, Balabaud $C$ et al. Nomenclature of the finer branches of the biliary tree: canals, ductules, and ductular reactions in human livers. Hepatology 2004; 15: 1739-1745.

21. Seki H, Ikeda F, Nanba S. Aberrant Expression of Keratin 7 in Hepatocytes as a Predictive Marker of Rapid Progression to Hepatic Failure in Asymptomatic Primary Biliary Cirrhosis. Acta Med Okayama 2015; 69 (3): 137-144.

22. Goldstein NS, Soman A, Gordon SC. Portal tract eosinophils and hepatocyte cytokeratin 7 immunoreactivity helps distinguish early-stage, mildly active primary biliary cirrhosis and autoimmune hepatitis. Am J Clin Pathol 2001; 116 (6): 846-853. 